\title{
Carrier Diffusion in the Barrier Enabling Formation of Charged Excitons in InAs/GaAs Quantum Dots
}

\author{
K.F. Karlsson* , E.S. Moskalenko ${ }^{\dagger}$, P.O. Holtz, \\ B. Monemar \\ Department of Physics and Measurement Technology, Linköping University \\ 58183 Linköping, Sweden
}

\author{
W.V. Schoenfeld, J.M. Garcia and P.M. Petroff \\ Materials Department, University of California - Santa Barbara \\ Santa Barbara, California 93106, USA
}

\begin{abstract}
It is demonstrated that the photoluminescence spectra of single self-assembled quantum dots are very sensitive to the experimental conditions, such as excitation energy and crystal temperature. A qualitative explanation is given in terms of the effective diffusion of the photogenerated carriers, determined by the experimental conditions, which influence the capture probability and hence also the charge state of the quantum dots. This is proposed as a new tool to populate quantum dots with extra electrons in order to study phenomena involving charged excitons.
\end{abstract}

PACS numbers: 78.67.Hc, 71.35.-y, 78.55.Cr

\section{Introduction}

Quantum dots (QDs) confine carriers in all three directions on the nanometer length scale. This implies a zero-dimensional (0D) density of states, similar to atoms, which is believed to improve the performance of electro-optical applications such as semiconductor lasers. In addition, the strong confinement results

*corresponding author; e-mail: freka@ifm.liu.se

${ }^{\dagger}$ Permanent address: A.F. Ioffe Physical-Technical Institute, Russian Academy of Sciences, 194021, Polytechnicheskaya 26, St. Petersburg, Russia. 
in an increased interaction between the trapped carriers, which also makes QDs interesting for single-electron devices. Consequently, multiparticle complexes in QDs are important to understand because they will influence on the performance of QD-based devices.

Multiparticle complexes include multiexcitons and charged excitons. Charged excitons in QDs have recently been studied theoretically [1-3] and experimentally in large ensembles [3]. However, the inhomogeneous broadening present in measurements of QD ensembles, caused by the inevitable size distribution, conceals features corresponding to the charging effects on the $\approx$ meV level. Very recently, experimental studies of the optical properties of charged excitons in single 0D systems were published, e.g. [4-6].

Two approaches have been used to control the charge state of 0D systems, both requiring special sample design. One method is to make use of a uniform background doping in the barrier, whereby the QD becomes loaded with a random number of excess carriers depending on the number of dopants in the near vicinity of the QD. The number of electrons or holes in the QD can then be controlled by means of photodepletion [4]. The other method is to use contacted structures with modulation doped layers [5, 6]. An external applied bias is then used to control the number of electrons.

The present paper is devoted to the creation of charged excitons in single InAs/GaAs QDs by purely optical means, relying on the different diffusivity of the photogenerated electrons and holes in the barrier. This can be used as a new effective tool to create and study charged excitons.

\section{Samples and experimental setup}

The samples studied were grown by molecular beam epitaxy on semi-insulating GaAs (100) substrates. On top of a $100 \mathrm{~nm}$ GaAs layer self-assembled lens-shaped QDs were formed on a wetting layer (WL) from about 1.7 ML InAs deposition. After special growth techniques to reduce the dot size [7] and size distribution, the QDs were covered by $100 \mathrm{~nm}$ GaAs for protection. A certain sample region with a low InAs deposition, and therefore a very low dot density [8], was chosen for our study where the interdot spacing was estimated to be about $10 \mu \mathrm{m}$, well exceeding the resolving power of the micro-photoluminescence setup (micro-PL setup) used.

The QDs were studied by means of a conventional diffraction-limited micro-PL setup. To excite the QDs, an Ar laser pumped by cw Ti-sapphire (Ti-Sp) laser, tunable between 700 and $900 \mathrm{~nm}$, was used with an excitation power adjusted by using relevant neutral filters. The beam of the Ti-Sp laser was focused on the sample surface by a microscope objective through a thin optical window of the continuous flow cryostat. The temperatures used in the experiments were ranging from $4 \mathrm{~K}$ to $40 \mathrm{~K}$. The laser beam could be focused on the sample surface down 
to a spot of diameter $2 \mu \mathrm{m}$. The luminescence signal was collected by the same objective and dispersed by a single-grating $0.45 \mathrm{~m}$ monochromator combined with a liquid $\mathrm{N}_{2}$ cooled Si-CCD camera. The spectral resolution achieved in the region of the studied PL was $0.15 \mathrm{meV}$.

A magnified image of the sample surface was projected on a video camera, allowing precise control of the laser position and focusing. A slight change in the temperature causes sample drift. Therefore special grids on the sample were used as a reference to locate the desired dot. The grids did not influence the QD spectra.

\section{Experimental results and discussion}

\subsection{Peak identification}

Several QDs have been studied, but most of them exhibit a similar behavior and are well represented by the spectra of the QD shown in the present paper. A typical low-temperature PL spectrum of a single QD is shown in Fig. 1a, taken at a moderate excitation power $\left(P_{\mathrm{ex}}=42 \mathrm{nW}\right)$ with the excitation energy ( $\left.\hbar \omega_{\text {photon }}\right)$ above the barrier band gap $\left(E_{\mathrm{g}}=1.519 \mathrm{eV}\right)$. The spectrum consists of two groups of emission lines, separated by $\approx 30 \mathrm{meV}$, denoted $s s$ and $p p$, respectively. At low and moderate excitation powers $\left(P_{\mathrm{ex}}<100 \mathrm{nW}\right)$, the integrated intensity of the $s s(p p)$ group exhibits linear (superlinear) dependence on the excitation power. At high power, the $p p$ intensity exceeds the $s s$ intensity, which is saturated. On the basis of this characteristic power dependence, the group $s s(p p)$ is attributed to transitions related to the ground states (first excited states) of the QD, i.e. transitions between electrons and holes, both in the $s(p)$ shells. This is also in consistence with the calculated energy separation between the $s s$ and $p p$ transitions, for lens-shaped QDs of similar composition and size [1].

This paper is mainly focused on the ss transitions, which exhibit a dramatic dependence on the excitation energy, in the case of $\hbar \omega_{\text {photon }}>E_{\mathrm{g}}$. Figures $1 \mathrm{~b}$ and c show two spectra of the ss emission of the same QD at two different excitation energies separated by $\approx 20 \mathrm{meV}$. Three peaks, denoted $X, X^{-}$, and $X^{--}$, are present in both spectra but with a remarkably different intensity distribution for the $X$ and $X^{--}$emissions. All three lines are present at the lowest excitation power and grow linearly with the power, without any intensity redistribution. Consequently, the peaks are not related to multiexciton complexes. However, the power dependence is consistent with charged complexes involving one exciton in the QD ground state [9]. To single out the peak corresponding to the neutral ground state exciton, the sample was excited with an energy below the WL, at which the electrons and holes are absorbed either into the QD directly or into localized states of the WL, whereby the population of the QD with an equal number of electrons and holes is expected. As shown in Fig. 1d, only the $X$ peak is present in the spectrum taken with excitation below the WL excitation, which therefore is ascribed to the neutral exciton. 


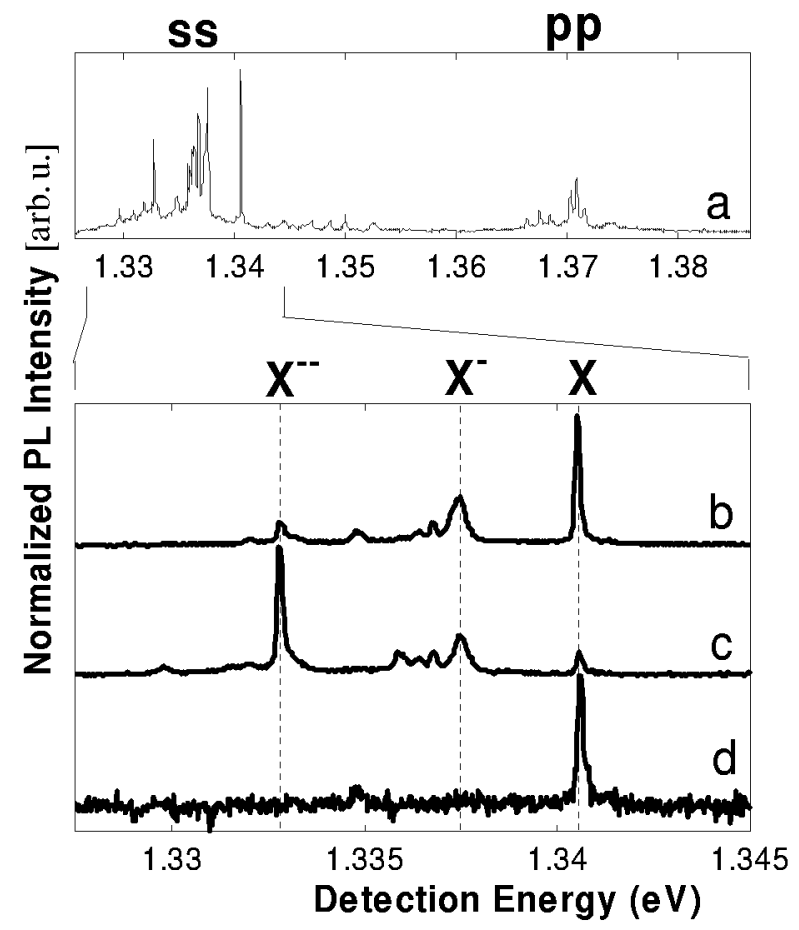

Fig. 1. Typical PL spectra of a single QD at temperature of $4 \mathrm{~K}$ for different excitation energies above the barrier band gap: (a) $\hbar \omega_{\text {photon }}=1.536 \mathrm{eV}\left(P_{\mathrm{ex}}=42 \mathrm{nW}\right)$, (b) $\hbar \omega_{\text {photon }}=1.561 \mathrm{eV}\left(P_{\mathrm{ex}}=10 \mathrm{nW}\right),\left(\right.$ c) $\hbar \omega_{\text {photon }}=1.542 \mathrm{eV}\left(P_{\mathrm{ex}}=10 \mathrm{nW}\right)$ and (d) below the barrier and below the WL, $\hbar \omega_{\text {photon }}=1.433 \mathrm{eV}\left(P_{\mathrm{ex}}=30000 \mathrm{nW}\right)$.

Calculations performed on lens-shaped QDs show that charged excitons, i.e. QDs with an extra electron (hole), in addition to the exciton, are red-shifted (blue-shifted) relative to the neutral exciton PL emission [10]. Since the peaks $X^{-}$ and $X^{--}$are red-shifted with respect to $X$ it is reasonable to interpret these peaks as negatively charged excitons, i.e. the QD is filled with extra electron(s). In order to estimate the binding energies of negatively charged excitons, a simple perturbation theory model developed in Ref. [3] was applied. The model assumes a parabolic lateral confining potential and a step-like potential in the growth direction, which has been shown to mimic the potential of self-assembled QDs very well [1]. Using the characteristic potential parameters for electrons and holes $\left(\hbar \omega_{\mathrm{e}}\right.$ and $\left.\hbar \omega_{\mathrm{h}}\right)$ as input, it is possible to obtain values of all interesting energies, i.e. the repulsion $E_{s s}^{\mathrm{c}}$ (attraction $E_{s s}^{\mathrm{eh}}$ ) between the $s$-electrons (the $s$-hole and the $s$-electron) and the exchange interaction $E_{s p}^{\mathrm{x}}$ between the $s$-electron and the p-electron. When an extra electron is added to the QD (which is already occupied with an exciton), two new interactions appear. The repulsion with the electron $E_{s s}^{c}$ and the attraction to the hole $E_{s s}^{\text {eh }}$ tend to increase and decrease the binding energy, respectively. The difference $E_{s s}^{\mathrm{eh}}-E_{s s}^{\mathrm{c}}$ gives the binding energy of the charged exciton with respect to 
the neutral exciton. Since the $s$-shell at maximum can accommodate two electrons, the addition of a third electron must result in population of the $p$-shell. This can be accomplished in two ways. The spin can be antiparallel or parallel to the spin of the $s$-electron forming the exciton. The former situation does not essentially change the binding energy from the case with one extra electron, while the latter situation gives rise to a further shift corresponding to $E_{s s}^{\mathrm{c}}[2]$.

The potential parameters used in the calculation are estimated to be $\hbar \omega_{\mathrm{e}} \approx$ $20 \mathrm{meV}$ and $\hbar \omega_{\mathrm{h}} \approx 10 \mathrm{meV}$, respectively, using the experimentally obtained energy difference of $\approx 30 \mathrm{meV}$ between the $s s$ and $p p$ transitions ${ }^{\ddagger}$. Using the effective masses and the dielectric constant deduced for similar QDs in Ref. [3], the binding energy of one extra electron is obtained to $2.6 \mathrm{meV}$ and the exchange energy is obtained to $E_{s s}^{\mathrm{c}}=4.9 \mathrm{meV}$. Comparing these values with the experimental values extracted from Fig. $1, E_{X}-E_{X-}=3.1 \mathrm{meV}$ and $E_{X-}-E_{X--}=4.7 \mathrm{meV}$, where $E_{P}$ correspond to the spectral position of peak $P$, indicates that $X^{-}\left(X^{--}\right)$corresponds to the charged exciton, i.e. the single exciton together with one (two) extra electrons. It should be noted that this good agreement between the experimental and the theoretical values is reproduced for other QDs studied as well.

\subsection{Influence of carrier diffusion}

In order to further investigate the dependence of the QD spectrum on the excitation energy, $\hbar \omega_{\text {photon, }}$, the PL spectrum was measured at different energies in the range from $1.52 \mathrm{eV}$ to $1.70 \mathrm{eV}$, i.e. above the barrier band gap. The intensities of $X$ and $X^{--}$exhibit a clear anticorrelated oscillating behavior with respect to $\hbar \omega_{\text {photon, }}$ as shown in Fig. 2. A similar competition between a neutral and a charged exciton has been reported previously for excitation in the WL [9].

The dashed vertical lines in Fig. 2 correspond to the energies $E_{\mathrm{g}}+n \hbar \omega_{\mathrm{LO}}$ $\times\left(1+m_{e}^{*} / m_{\mathrm{h}}^{*}\right)$, where $n$ is an integer $(n=1,2,3$, or 4$), \hbar \omega_{\mathrm{LO}}=36 \mathrm{meV}$ is the GaAs LO-phonon energy and $m_{\mathrm{e}}^{*}=0.067 m_{0}\left(m_{\mathrm{h}}^{*}=0.45 m_{0}\right)$ is the electron (heavy-hole) effective mass in GaAs $[11,12]$. Consequently, these energy values correspond to the excitation energies $\hbar \omega_{\text {photon }}$ at which the electron's part of the excess energy in GaAs is exactly an integer times $\hbar \omega_{\mathrm{LO}}$, assuming parabolic band dispersion [14]. It should be noted that the lines coincide with the intensity maxima (minima) of the $X\left(X^{--}\right)$peak.

The oscillating dependence of the QD exciton intensity on the excitation energy $\hbar \omega_{\text {photon }}$ can be explained in terms of the well-known phonon relaxation processes of carriers [13]. The excess energy of the photogenerated carriers is mainly released either by fast [12] (few ps) emission of LO-phonons or by much slower emission of acoustic phonons. If the excess energy exactly fits with an integer

$\ddagger$ As follows from the solution of the simple two-dimensional harmonic oscillator, the ratio $\hbar \omega_{\mathrm{h}} / \hbar \omega_{\mathrm{e}}$ depends only on the effective masses and the depths of the confining parabolic potentials. Therefore, $\hbar \omega_{\mathrm{h}} / \hbar \omega_{\mathrm{e}}$ is expected to be close to $1 / 2$ for the QDs studied in the present paper, as obtained both experimentally and theoretically for similar QDs $[3,1]$. 


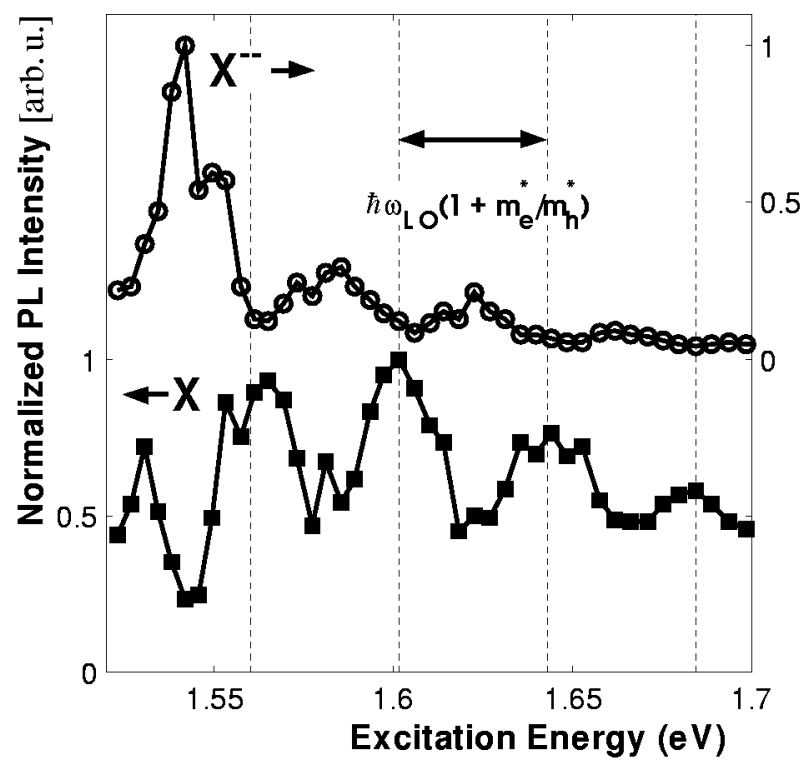

Fig. 2. The intensity dependence of the $X$ and $X^{--}$peaks in the QD ground state (ss) versus the above barrier band gap excitation energy, at temperature of $4 \mathrm{~K}$. The dashed lines correspond to the energies at which the excited electron's part of the excess energy is exactly an integer times the LO-phonon energy in the GaAs barrier.

number of $\hbar \omega_{\mathrm{LO}}$, the excess energy is very quickly released, whereby the carrier becomes motionless at the band edge shortly after generation. On the other hand, if the excess energy does not fit with an integer number of $\hbar \omega_{\mathrm{LO}}$, it takes much longer time to release the energy remaining after the LO-phonon emission. During this additional time the carrier is able to move a longer distance in the crystal. In other words, excess energies that fit with (do not fit) $\hbar \omega_{\mathrm{LO}}$ will result in a low (high) effective diffusivity. Carriers with higher diffusivity will move in a spatially larger region and are thus more likely to pass in the near vicinity of a strong capturing center (a quantum well or a QD) and become trapped. Due to different masses, the excess energy is distributed in a non-equal way between the electrons and the heavy holes, which also results in different diffusivities. In an initial state the capturing center will primarily trap the high diffusivity carriers, but subsequently the attraction of low diffusivity carriers of opposite charge will be increased by the Coulomb interaction. The steady-state condition at continuous excitation requires equal capture probability of electrons and holes. This condition is thus precisely fulfilled when capturing center accommodates a certain number of extra carriers of higher diffusivity.

Another way to increase the effective diffusivity of the carriers is to increase the crystal temperature $T$ [14]. Since the electron mass is about seven times lighter than the heavy-hole mass in GaAs, the electrons gain higher diffusivity 
than the heavy holes, when the temperature is increased. The PL spectra shown in Fig. 3 demonstrate the temperature dependence at three different excitation

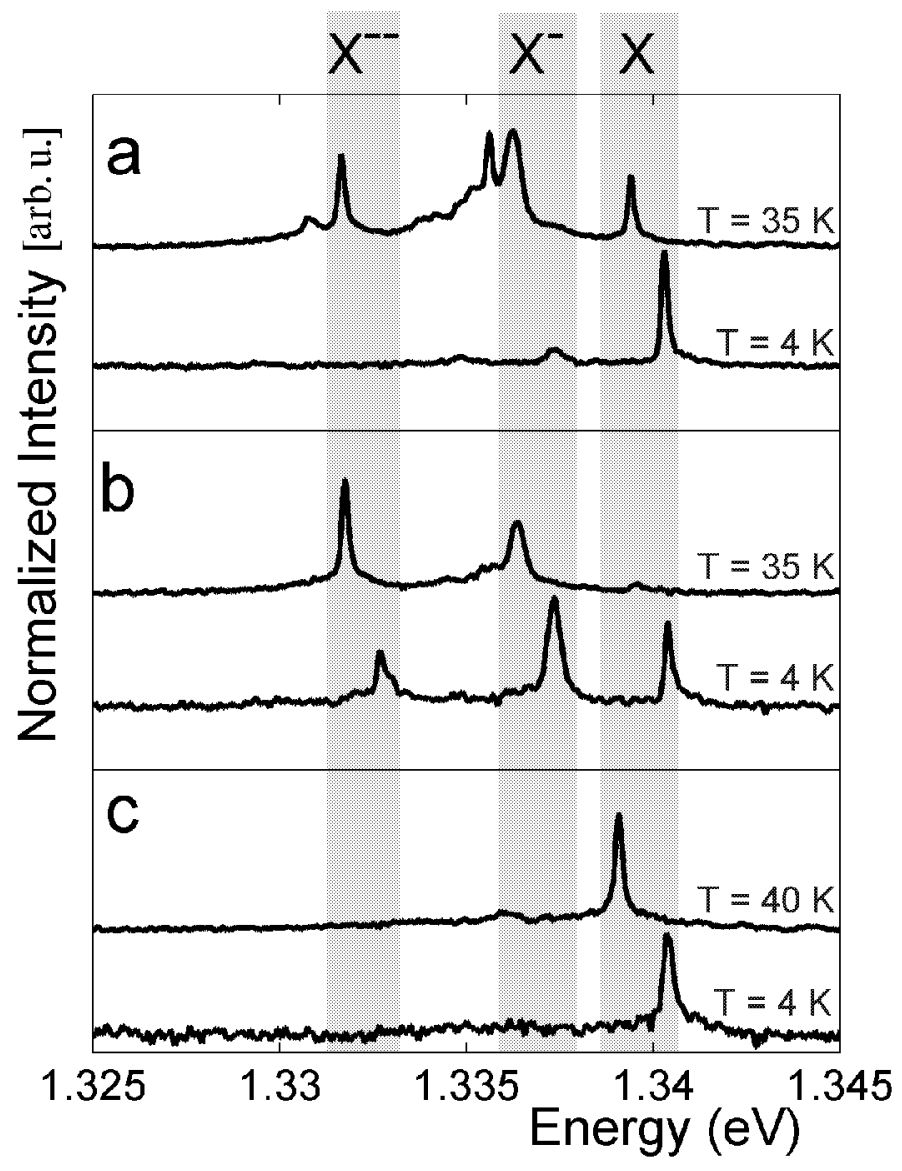

Fig. 3. The temperature influence on the QD ground state emission at excitation energies above the barrier band gap: (a) $\hbar \omega_{\text {photon }}=1.686 \mathrm{eV}\left(P_{\mathrm{ex}}=5 \mathrm{nW}\right)$ and (b) $\hbar \omega_{\text {photon }}=1.557 \mathrm{eV}\left(P_{\mathrm{ex}}=5 \mathrm{nW}\right)$ and below the barrier and below the WL, (c) $\hbar \omega_{\text {photon }}=1.433 \mathrm{eV}\left(P_{\mathrm{ex}}=30000 \mathrm{nW}\right)$. 
energies, $\hbar \omega_{\text {photon }}$. To maintain the same excess energy for the spectra taken at $\hbar \omega_{\text {photon }}>E_{\mathrm{g}}$ at both temperatures $T=4 \mathrm{~K}$ and $T=35 \mathrm{~K}$, the excitation energy at $T=35 \mathrm{~K}$ was slightly shifted $(<0.5 \mathrm{meV})$ from its value at $T=4 \mathrm{~K}$ to follow the thermal shift of the GaAs band gap. An analogous shift is also observed for the QD luminescence, as can be seen in Fig. 3. In Fig. 3a, the excitation energy $\hbar \omega_{\text {photon }}$ is chosen to give solely the pure exciton $X$ at $4 \mathrm{~K}$. When the temperature is increased, there is a successive transfer of the intensity from $X$, initially to $X^{-}$, and subsequently also to $X^{--}$. At $35 \mathrm{~K}$ the three lines have comparable intensity. In Fig. 3b, the excitation energy $\hbar \omega_{\text {photon }}$ is chosen to give comparable intensities for the three peaks at $4 \mathrm{~K}$. At $35 \mathrm{~K}$ the corresponding spectrum is dominated by $X^{--}$, while $X$ has vanished completely. The observed monotonous decrease in $X$ and the corresponding increase in $X^{--}$indicates that an increased temperature effectively fills the QD with extra electrons.

To finally confirm that the temperature effect is due to an increased diffusivity and not due to an activation of localized electrons (for example electrons localized at impurities caused by unintentional background doping) the sample was excited with an excitation energy $\hbar \omega_{\text {photon }}$ below the WL emission, while the temperature was increased (Fig. 3c). Since the photogenerated carriers in this case are localized and hence are totally immobile, no diffusion can take place. Accordingly no peak except the pure exciton $X$ is expected in the spectrum. If the extra electrons, on the other hand, are due to electron activation, the spectrum should qualitatively exhibit the same temperature behavior as for excitation energies $\hbar \omega_{\text {photon }}>E_{\mathrm{g}}$. Obviously, the spectra in Fig. $3 c$ are temperature independent, except for a small red shift with the same amount as monitored in Figs. 3a and $3 \mathrm{~b}$, which is fully consistent with the explanation in terms of diffusivity.

\section{Conclusions}

In conclusion, three peaks in the QD ground state have been identified as the neutral, single charged and double charged exciton, respectively, in good agreement with a simple theoretical model. It has been shown that the QD spectrum is very sensitive to the excitation energy above the barrier band gap and the crystal temperature, since these parameters are shown to effectively influence the charge state of the QD. This is an important fact and should always be taken into account when performing non-resonant excitation on the QDs. Furthermore, the sensitivity on the excitation energy is qualitatively explained in terms of the well known phonon relaxation processes, which periodically modulate the effective diffusivity of the photogenerated carriers and consequently also the initial capture probability of the QD. This can be used as a new effective tool to create and study phenomena involving charged excitons by purely optical means, without special sample design or doping. 


\section{Acknowledgments}

One of us (E.S.M.) gratefully acknowledges financial support of the Swedish Institute within the Visby Programme.

\section{References}

[1] A. Wojs, P. Hawrylak, S. Fafard, L. Jacak, Phys. Rev. B 54, 5604 (1996).

[2] P. Hawrylak, Phys. Rev. B 60, 5597 (1999); A. Wojs, P. Hawrylak, Phys. Rev. B 55, 13066 (1997).

[3] R.J. Warburton, B.T. Miller, C.S. Durr, C. Bodefeld, K. Karrai, J.P. Kotthaus, G. Medeiros-Ribeiro, P.M. Petroff, S. Huant, Phys. Rev. B 58, 16221 (1998).

[4] A. Hartmann, Y. Ducommun, E. Kapon, U. Hohenester, E. Molinari, Phys. Rev. Lett. 84, 5648 (2000); A. Hartmann, Y. Ducommun, M. Bachthold, E. Kapon, Physica E 7, 461 (2000).

[5] R.J. Warburton, C. Shäflein, D. Haft, F. Bickel, A. Lorke, K. Karraö, J.M. Gracia, W. Schoenfeld, P.M. Petroff, Nature 405, 926 (2000).

[6] F. Findeis, M. Baier, A. Zrenner, M. Bichler, G. Abstreiter, U. Hohenester, E. Molinari, Phys. Rev. B 63, 121309 (2001).

[7] J.M. Garcia, T. Mankad, P.O. Holtz, P.J. Wellman, P.M. Petroff, Appl. Phys. Lett. 72, 3172 (1998).

[8] D. Leonard, K. Pond, P.M. Petroff, Phys. Rev. B 50, 11687 (1994).

[9] J.J. Finley, A.D. Ashmore, A. Lemaître, D.J. Mowbray, M.S. Skolnick, E.I. Itskevich, P.A. Maksym, M. Hopkinson, T.F. Krauss, Phys. Rev. B 63, 073307-1 (2001).

[10] Ph. Lelong, G. Bastard, Solid. State Commun. 98, 819 (1996).

[11] J.P. Wolfe, H.W. Yoon, D.R. Wake, H. Morkoc, Semicond. Sci. Technol. 7, B240 (1992).

[12] H.W. Yoon, D.R. Wake, J.P. Wolfe, Phys. Rev. B 54, 2763 (1996).

[13] R.W. Shaw, Phys. Rev. B 50, 3283 (1971); A. Nakamura, C. Weisbuch, Solid-State Electron. 21, 1331 (1978).

[14] H.W. Yoon, D.R. Wake, J.P. Wolfe, Phys. Rev. B 46, 13461 (1992); H. Hillmer, A. Forchel, S. Hansmann, M. Morohashi, E. Lopez, H.P. Meier, K. Ploog, Phys. Rev. B 39, 10901 (1989). 